\title{
Improving Free-Piston Stirling Engine Specific Power
}

\author{
Maxwell H. Briggs ${ }^{1}$ \\ NASA Glenn Research Center, Cleveland, Ohio,44135
}

\begin{abstract}
This work uses analytical methods to demonstrate the potential benefits of optimizing piston and/or displacer motion in a Stirling Engine. Isothermal analysis was used to show the potential benefits of ideal motion in ideal Stirling engines. Nodal analysis is used to show that ideal piston and displacer waveforms are not optimal in real Stirling engines. Constrained optimization was used to identify piston and displacer waveforms that increase Stirling engine specific power.
\end{abstract}

\section{Introduction}

$\mathrm{F}$ ree-piston Stirling devices are closed-cycle regenerative devices that can achieve high efficiencies $(>50 \%$ of Carnot efficiency is achievable in well-made engines). Applications include cryo-coolers, natural gas cogeneration units, solar-dynamic power conversion, and nuclear dynamic power conversion. They are typically used in applications which benefit from high efficiency or in systems that require closed cycle operation. High efficiency and closed-cycle operation are both requirements of many space power systems, making free-piston Stirling engines excellent candidates for these applications. They are being considered for power conversion in NASA missions that require Radioisotope Power Systems because their high efficiency allows NASA to use less of the limited supply of plutonium-238 per mission. They also trade favorably in some fission power applications because their high efficiency requires less heat input from the reactor and reduces heat rejection requirements, which reduces the mass of the reactor shield and the radiators ${ }^{1}$. Stirling engines have been considered for use in several terrestrial applications including automotive engines, solar dish-Stirling power plants, and residential co-generation systems, especially when rising fossil fuel costs increase the cost benefit of their high efficiency. However, the low specific power of Stirling engines typically prevent them from competing with internal combustion engines when fuel costs are low and open-cycle engines are viable.

One potential method of increasing Stirling engine specific power, and therefore its range of application, is to enforce piston and/or displacer motion that more closely approximate those of the ideal Stirling cycle. The ideal Stirling thermodynamic cycle consists of isothermal compression and expansion processes and constant volume heat addition and heat removal processes (Fig. 1).
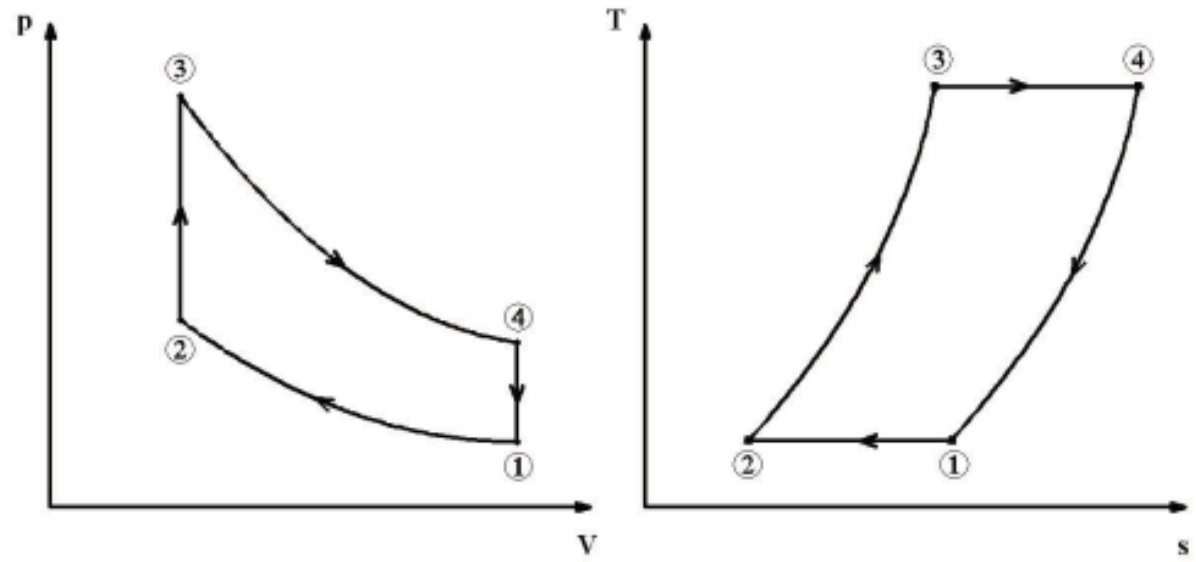

Figure 1. Ideal Stirling P-V and T-S Diagrams.

Achieving the ideal cycle requires that the piston and displacer dwell and abruptly change direction throughout the cycle, which has been difficult to achieve in both kinematic and free-piston configurations. Designers typically choose more practically achievable motion, most often converging on a mechanical linkage or electrical control scheme that imposes sinusoidal motion. While there are certainly benefits to sinusoidal motion, it is not necessarily the optimal choice from a performance perspective since sinusoidal motion may reduce specific power.

\footnotetext{
${ }^{1}$ Mechanical Engineer, Thermal Energy Control Branch, 21000 Brookpark Rd MS 301-1, AIAA Member
} 
Figure 2 illustrates the ideal piston and displacer motion of a Stirling engine in the beta configuration, as well as a plot of ideal piston and displacer position. Process 1-2 shows constant temperature compression, in which the displacer remains still and the piston compresses the gas as heat is removed through the cooler. Process 2-3 shows constant volume heat addition, in which the displacer moves gas from the cold side to the hot side, through the regenerator, while the piston remains still. Process 3-4 is a constant temperature expansion process, in which the expansion of the gas moves the piston and the displacer moves along with it to maximize the amount of gas in the expansion space. The work done during the expansion process minus the work done during the compression process is the usable power of the Stirling cycle. In free-piston engines this power is extracted through the linear alternator. Process $4-1$ is a constant volume heat removal process in which the displacer moves gas from the hot to the cold side, through the regenerator, while the piston remains still.
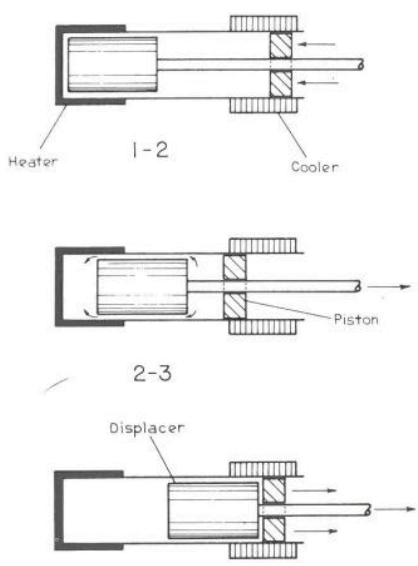

3-4

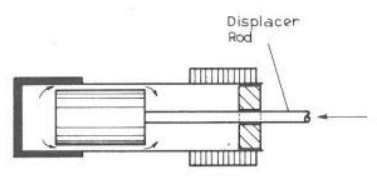

4-1

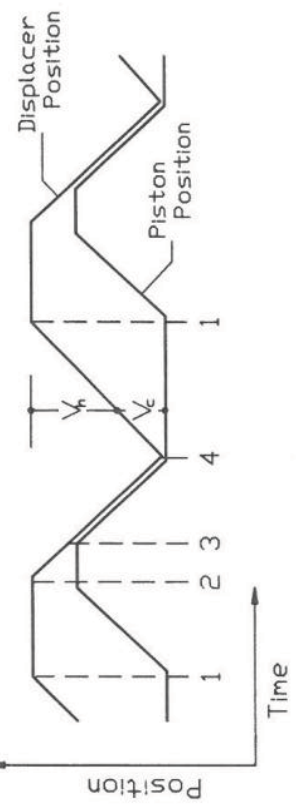

Figure 2. Schematic and plots of ideal piston and displacer motion. Figure from Ref. 2

\section{Isothermal Analysis of Highly Idealized Engines}

The classical analysis of Stirling engines resulting in a closed-form solution assumes the following:

1. Gas volumes are isothermal at either the hot-end temperature or the cold-end temperature or the regenerator temperature (No unswept volume).

2. The instantaneous pressure is constant throughout the working space

3. Working fluid behaves as an ideal gas with constant specific heat

4. No leakage of working fluid between gas volumes

5. The regenerator has a linear temperature profile that is constant in time

The more often used Schmidt analysis adds the additional assumption that the expansion and compression pistons move sinusoidally, with a phase shift, $\alpha$, and also results in a closed form solution. A complete derivation of the ideal Stirling and Schmidt cycle work can be found in Ref. 2, and is widely available in the literature. The relavent conclusions of the ideal Stirling and Schmidt cycle analysis appear below:

$$
\begin{gathered}
\mathrm{W}_{\text {net,ideal }}=\mathrm{M}_{\text {tot }} \mathrm{RT}_{\mathrm{CS}}[\tau-1] \ln \left(\frac{\mathrm{r}+\mathrm{d}}{1+\mathrm{d}}\right) \\
\mathrm{r}=\frac{\mathrm{V}_{\text {max }}}{\mathrm{V}_{\text {min }}} \quad \mathrm{\tau}=\frac{\mathrm{T}_{\mathrm{ES}}}{\mathrm{T}_{\mathrm{CS}}} \quad \mathrm{d}=\frac{\mathrm{C}_{1}}{\mathrm{~V}_{\text {min }}} \\
\mathrm{C}_{1}=\mathrm{T}_{\mathrm{CS}}\left(\frac{\mathrm{V}_{\text {Unswept }}}{\mathrm{T}_{\mathrm{CS}}}+\frac{\mathrm{V}_{\mathrm{C}}}{\mathrm{T}_{\mathrm{C}}}+\frac{\mathrm{V}_{\mathrm{R}}}{\mathrm{T}_{\mathrm{Eff} \mathrm{R}}}+\frac{\mathrm{V}_{\mathrm{H}}}{\mathrm{T}_{\mathrm{H}}}+\frac{\mathrm{V}_{\text {Unswept }}}{\mathrm{T}_{\mathrm{ES}}}\right)
\end{gathered}
$$




$$
\begin{gathered}
\mathrm{W}_{\text {net,Schmidt }}=\frac{2 \pi \mathrm{M}_{\mathrm{tot}} \mathrm{RT}_{\mathrm{ES}}}{(\tau+\kappa)}\left(\frac{\sqrt{1-\mathrm{b}^{2}}-1}{\mathrm{~b} \sqrt{1-\mathrm{b}^{2}}}\right)(\sin (\beta)-\kappa \sin (\beta-\alpha)) \quad(2) \\
\beta=\tan ^{-1}\left(\frac{\kappa \sin \alpha}{\kappa \cos \alpha+\tau}\right) \quad \mathrm{\kappa}=\frac{\mathrm{V}_{\mathrm{ES}, \mathrm{sw}}}{\mathrm{V}_{\mathrm{CS}, \mathrm{sw}}} \quad \mathrm{b}=\frac{\frac{\mathrm{V}_{\mathrm{CS}, \mathrm{sw}}}{2 \mathrm{~T}_{\mathrm{ES}}} \sqrt{\tau^{2}+2 \kappa \tau \cos (\alpha)+\kappa^{2}}}{\mathrm{C}_{2}} \\
\mathrm{C}_{2}=\left(\frac{\mathrm{V}_{\mathrm{CS}, \mathrm{sw}}}{2 \mathrm{~T}_{\mathrm{CS}}}+\frac{\mathrm{V}_{\mathrm{CS}, \text { clearance }}}{\mathrm{T}_{\mathrm{CS}}}+\frac{\mathrm{V}_{\mathrm{C}}}{\mathrm{T}_{\mathrm{C}}}+\frac{\mathrm{V}_{\mathrm{R}}}{\mathrm{T}_{\mathrm{Eff} \mathrm{R}}}+\frac{\mathrm{V}_{\mathrm{H}}}{\mathrm{T}_{\mathrm{H}}}+\frac{\mathrm{V}_{\mathrm{ES}, \text { clearance }}}{\mathrm{T}_{\mathrm{ES}}}+\frac{\mathrm{V}_{\mathrm{ES}, \mathrm{sw}}}{2 \mathrm{~T}_{\mathrm{ES}}}\right)
\end{gathered}
$$

In general, ideal Stirling cycle work is a function of the temperature ratio, volume ratio, and dead volume ratio. Schmidt cycle work is a function of temperature ratio, phase angle, swept volume ratio (not to be confused with the volume ratio), and the parameter $\frac{C_{2} T_{E S}}{V_{C S, S w}}$. In order to compare the specific power of the ideal cycle and Schmidt cycle it is useful to take the work ratio of the two cycles. In the most general case the work ratio is a function of six independent dimensionless parameters. To simplify comparisons, this analysis considers the special case of zero dead, unswept, and clearance volumes. The assumptions used for this analysis are not representative of real engines and the shortcomings of these assumptions will be discussed in later sections, but starting from a generalized closedform solution on an idealized engine provides a useful illustration of the fundamentals of the relationship between Stirling engine specific power and piston/displacer motion.

A choice must also be made about how relate the sinusoidal piston motion of the Schmidt cycle to the ideal Stirling piston motion in a way that gives useful comparisons. The appropriate choice of constraints depends on the operational constraints of an individual engine. One can argue that the ideal and Schmidt engines should be constrained to have common maximum and minimum working space volumes, so that power differences are not the result of differences in working space volume amplitude. Using this approach, increased specific power of the ideal cycle is solely the result of holding the working space at constant volume during heat addition and removal. It does not take into account the fact that, for the same limits of piston motion, the ideal cycle can achieve higher working space volume amplitude. Alternatively the Schmidt and Stirling cycles could be constrained to have common limits of piston and displacer motion, allowing the ideal cycle to achieve higher working space volume amplitude. Note that there are several other possible choices for constraints which are not considered (i.e. setting maximum pressure of the Schmidt and ideal cycles equal or constraining the out limits of piston and displacer motion and leaving the in limit unconstrained).

Using common values for the working space volume amplitude and forcing the ideal cycle to operate at lower expansion and compression volume amplitude than the Schmidt cycle gives the following relationship for the ideal working space volume ratio, $r$, in terms of $k$ and $\alpha$ :

$$
r=\frac{(\kappa+1)+\sqrt{\kappa^{2}+2 \kappa \cos (\alpha)+1}}{(\kappa+1)-\sqrt{\kappa^{2}+2 \kappa \cos (\alpha)+1}}
$$

Substituting Eq. 3 into Eq. 1 and taking the ratio of ideal cycle work to Schmidt cycle work shows the increase in specific power for engines operating with ideal piston. These results are plotted in Fig. 3 for several values of $\mathrm{k}, \tau$, and $\alpha$. 

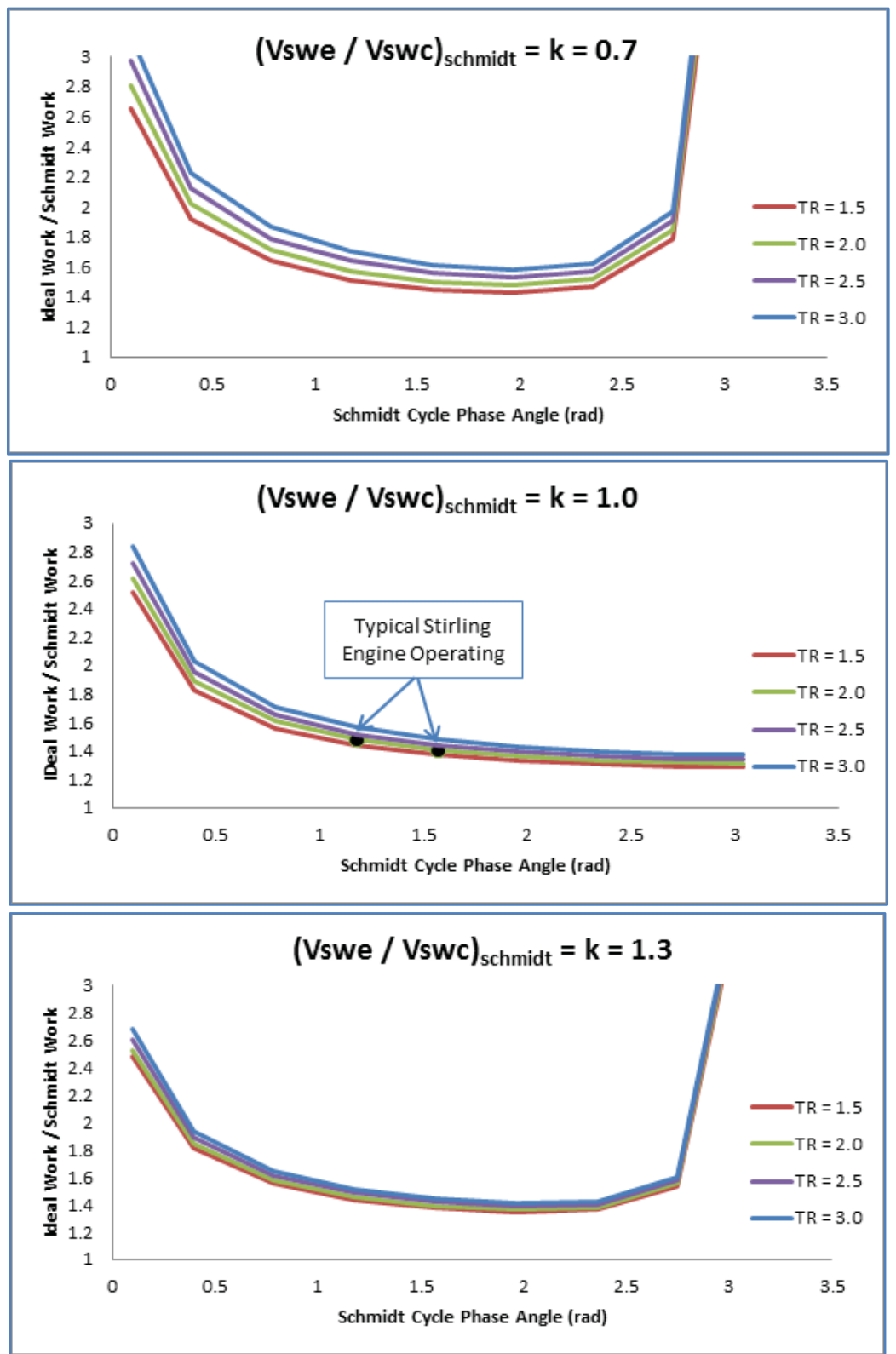

Figure 3. Ratio of ideal cycle work to Schmidt cycle work assuming both cycles have equal maximum working space volume and equal minimum working space volume.

The specific power improvement ranges from $28 \%$ to infinity depending on the operating conditions of the engine. The largest increases in specific power occur at impractically high or low phase angles where both the ideal and Schmidt cycles produce very little power. Figure 3 calls out two more typical Stirling engine operating points with a temperature ratio of 2 , swept volume ratio of 1 , and phase angle between 45 and 90 degrees. At these conditions the potential increase in specific power is between $37 \%$ and $56 \%$.

Another method of comparing specific power is to require the ideal cycle and Schmidt cycle to share common limits of piston and displacer motion, allowing the ideal cycle to run at higher working space stroke. Under this set of constraints the swept volume ratio of the Schmidt cycle, $\kappa$, is forced to unity and the ideal working space volume ratio, $r$, remains an independent variable, so work ratio becomes: 


$$
\frac{W_{\text {ideal }}}{W_{\text {Schmidt }}}=\frac{\ln (r)\left(\tau^{2}-1\right)}{2 \pi \tau\left(\frac{\sqrt{1-b^{2}}-1}{b \sqrt{1-b^{2}}}\right)[\sin \beta-k \sin (\beta-\alpha)]}
$$

This shows that the work ratio is proportional to $\ln (\mathrm{r})$, in theory ranging from negative infinity to positive infinity. However, running an ideal Stirling at a lower working space volume ratio than that of the equivalent Schmidt cycle would be counterproductive, so the Schmidt cycle working space volume ratio is the practical lower limit for ideal cycle volume ratio. Figure 4 shows the ratio of ideal work to Schmidt work as a function of $r$ for several values of $\tau$ and $\alpha$ where the minimum ideal cycle swept volume ratio is equal to the Schmidt cycle swept volume ratio. Figure 4 shows a specific power improvement of $32 \%$ at low volume ratios and shows the specific power improvement increasing without bound according to $\ln (\mathrm{r})$ as the volume ratio increases.

These two comparisons illustrate the differences in specific power that result from approximating ideal piston motion with sinusoidal piston motion. The ideal cycle has three advantages over the Schmidt cycle: 1) Constant volume heat addition/rejection in the ideal cycle increases the pressure ratio which increases specific power 2) dwelling periods of the ideal cycle allow it to achieve larger working space strokes, even when operating within the same limits of piston and displacer motion as the Schmidt cycle 3) dwelling pistons results in the minimum amount of hot gas being compressed and the minimum amount of cold gas being expanded. Using common working space volumes for the ideal cycle and Schmidt cycle eliminates the effect of benefit number two resulting in a specific power improvements ranging from $28 \%$ to infinity. Analyzing the effects of both constant volume heat addition/rejection and higher working volume ratios shows specific power improvement ranging from $32 \%$ to infinity, depending on the sorking space volume ratio of the ideal engine. These results show that using ideal piston motion could potentially increase specific power open a new trade space for
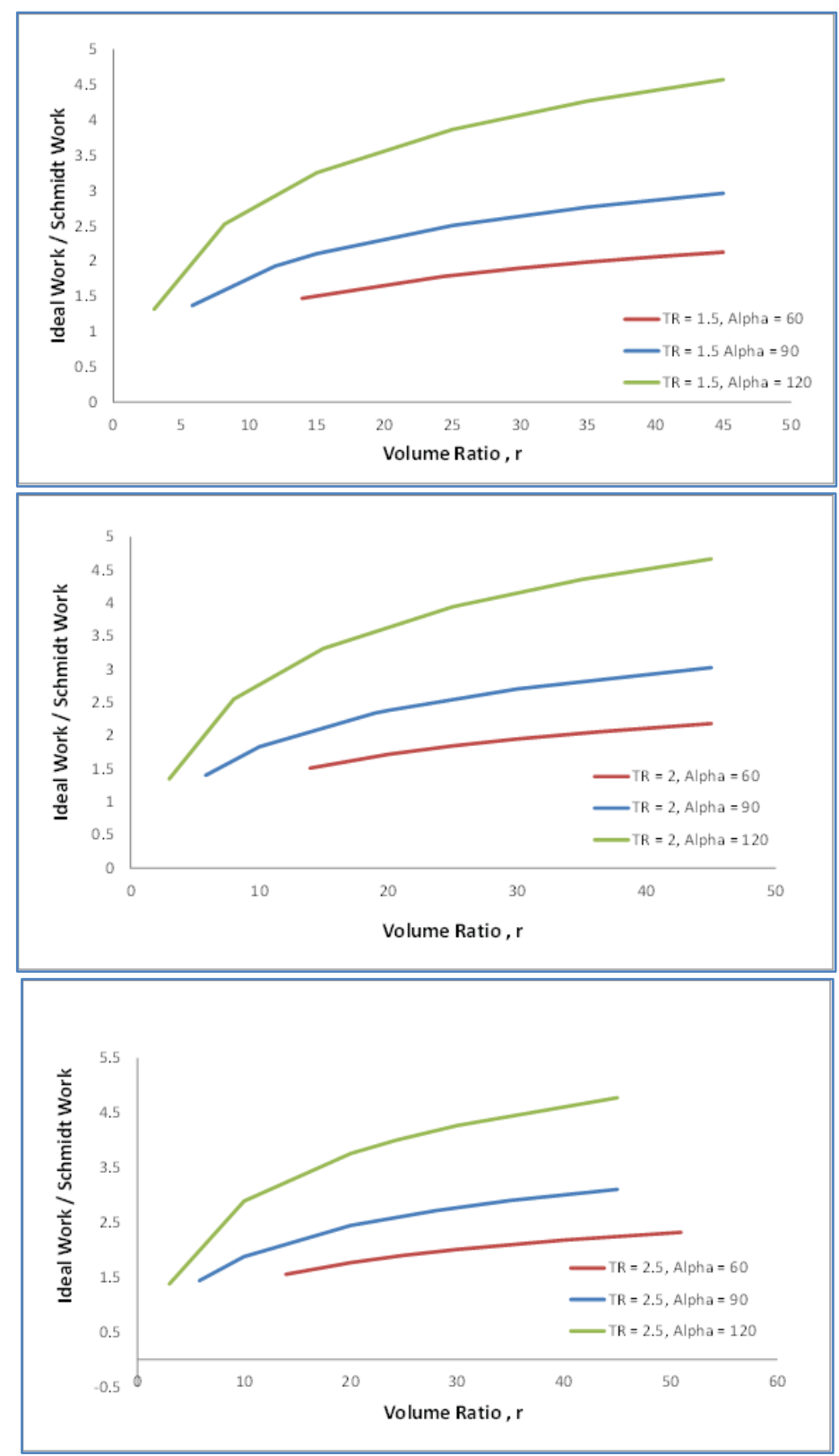

Figure 3. Ratio of ideal cycle work to Schmidt cycle work assuming equal maximum and minimum swept volumes. engine designers. However, this analysis is highly idealized and does not account for many aspects of real engines including dead volume, heat exchanger pressure drop, seal leakage, conduction losses, and other parasitics. The conclusions of this analysis are meant to show potential benefits in a simple and general way and should not be interpreted as an accurate prediction of specific power improvement on real engines. However, it is an encouraging first step to see that theory predicts the potential for large increases in specific power when going from the Schmidt cycle to the ideal cycle. 


\section{Nodal Analysis of Ideal Waveforms on a Realistic Geometry}

The preceding analysis considered a general and highly idealized Stirling engine to illustrate, as simply as possible, the potential advantages of using piston motion that more closely approximates ideal piston motion. However, several of the assumptions used in the preceding section depart from reality and undermine the usefulness of the results. The assumption of no dead volume allowed the ideal cycle to achieve unrealistically high pressure ratios when the volume ratio was treated as an independent variable. Real Stirling engines require dead volume in the heat exchangers and regenerator to achieve the required heat transfer. This dead volume limits the pressure ratio and specific power of the ideal cycle, but was not considered. Isothermal analysis also assumes that heat transfer rates in the expansion and compression spaces are so high that the temperature of the gas within those volumes can be considered isothermal. Under these conditions there is no need for a distinct heater or cooler, because all of the necessary heat transfer takes place in the expansion and compression spaces. Real engines lack sufficient surface area in the compression and expansion spaces to accomplish this, so high surface area heaters and coolers are added along with their associated dead volume. The assumptions of isothermal (and adiabatic) analysis both inaccurately model heat transfer in the the heater, cooler, expansion and compression spaces. The actual heat transfer in each gas space is a function of heat exchanger geometry and flow conditions and must be calculated to obtain accurate results. In addition, isothermal analysis does not take into account several potentially substantial loss mechanisms including pressure drop through the heat exchangers, leakage between the compression and expansion spaces, leakage between the working space and bounce space, and conduction from the hot end to the cold end.

Rectifying these shortcomings requires nodal analysis and detailed knowledge of engine geometry and a corresponding loss of generality in the solution. The nodal analysis tool used in this section is the commercially available Stirling analysis program Sage $\mathcal{C}$. Sage is commonly used for Stirling engine optimization in the design phase and performance mapping of engines once the design has been finalized. The engine chosen for analysis in this section is a 1-kW Stiurling engine designed for use in European cogeneration systems. The Sage model of the1$\mathrm{kW}$ engines is a nodal, one-dimensional, cyclic steady-state model that couples the equations of motion of the piston and displacer with the Navier-Stokes equations, and energy equations. Sage can also be used to calculate the reduction in available energy which is ignored by ideal, isothermal, and adiabatic analysis.

The Sage model of the 1-kW engines assumes an isothermal boundary condition on solid surfaces within the engine; it then calculates temperature gradients in the rest of the solid and the gas based on solid conduction and calculated convection coefficients. The base temperature of the finned hot-end heat exchanger (acceptor) is set as an input. These temperature inputs typically come from heat transfer analysis done outside of Sage. Sage then iteratively solves for gas temperatures and pressure drops by guessing and checking fin temperature profiles and gas velocities, and the resulting displacer motion (piston motion is typically a user input). Figure 4 shows a P-V diagram generated by Sage for the 1$\mathrm{kW}$ P2A engines. The P-V diagram generated from isothermal sinusoidal analysis is included for reference. The predicted power output for isothermal analysis is $40 \%$ larger than the Sage predicted power output. Isothermal analysis predicts an engine efficiency equal to the Carnot efficiency, which in this case is $0.550(\mathrm{Th}=779 \mathrm{~K}$ and $\mathrm{Tc}=350$ $\mathrm{K})$ which is $80 \%$ higher than the

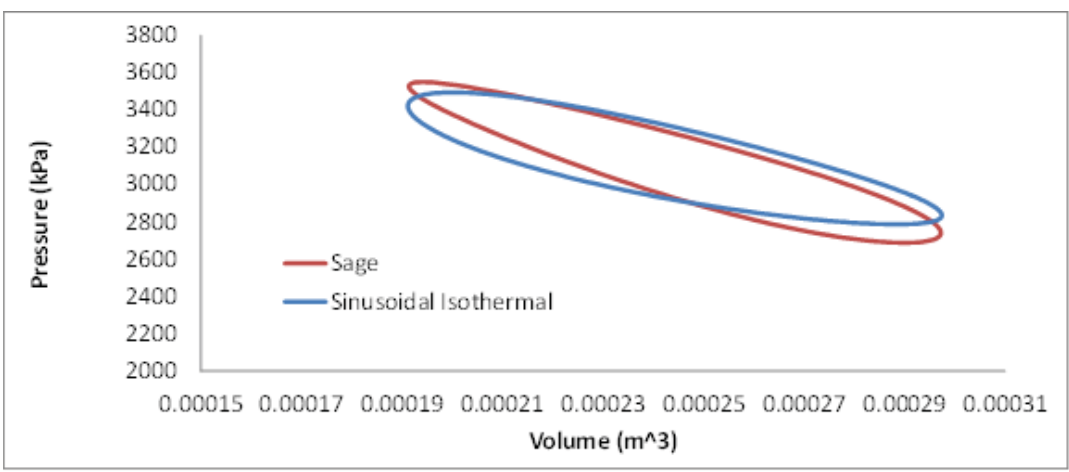

Figure 4. Comparison of P-V diagrams for a 1-kW Stirling engine using nodal analysis and Schmidt analysis efficiency of 0.307 predicted by Sage, suggesting that isothermal analysis inaccurately predicts the heat transfer requirements of real engines.

Sage requires all non-sinusoidal time-dependent inputs to be entered as sums of harmonic sinusoids. As higher harmonics are added the solver time step must be reduced to resolve the higher frequencies, increasing computational time. Ideal piston and displacer motion was approximated using 7-term truncated Fourier series. There is some overshoot (ringing) associated with using truncated Fourier series, but this effect was usually small since the quality of fit was high in all cases $\left(\mathrm{R}^{2}\right.$ values for all cases run were above 0.995$)$. 
One inherent problem in modeling ideal piston and displacer motion in Sage, or any other high fidelity Stirling model, is that there are an infinite combination of piston and displacer motions which are considered ideal. Figure 5 shows four different piston motions with varying piston dwell times, all of which satisfy the requirements of ideal piston and displacer motion. The top graph shows an extreme case of symmetric piston motion with long piston dwell times and infinite piston velocities. The second graph shows a cycle with symmetric piston motion, moderate dwell times, and moderate piston velocities. The third graph shows another extreme case of symmetric piston motion with piston dwell times of zero and the lowest possible piston velocities. The bottom picture shows a case of asymmetric piston motion with moderate dwell times and moderate piston velocities. Each example of ideal motion presented in Figure 5 is constrained to have the same minimum expansion volume, minimum clearance between the piston and displacer, maximum working space volume, and minimum working space volume as an equivalent engine undergoing sinusoidal motion. In addition to the waveforms shown in Figure 5 others can be generated using other constraints and even more can be generated if ideal displacer and piston velocities are not forced to be piecewiseconstant, as they have been shown to this point. Since isothermal analysis assumes that heat transfer takes place instantaneously and does not take into account pressure drop or other loss mechanisms that are functions of piston and displacer velocity, predicted power output and efficiency each of these cycles are equal, regardless of dwell times or piston/displacer velocities. However, in higher fidelity nodal analysis, insufficient dwell times limit working fluid temperatures and higher gas velocities
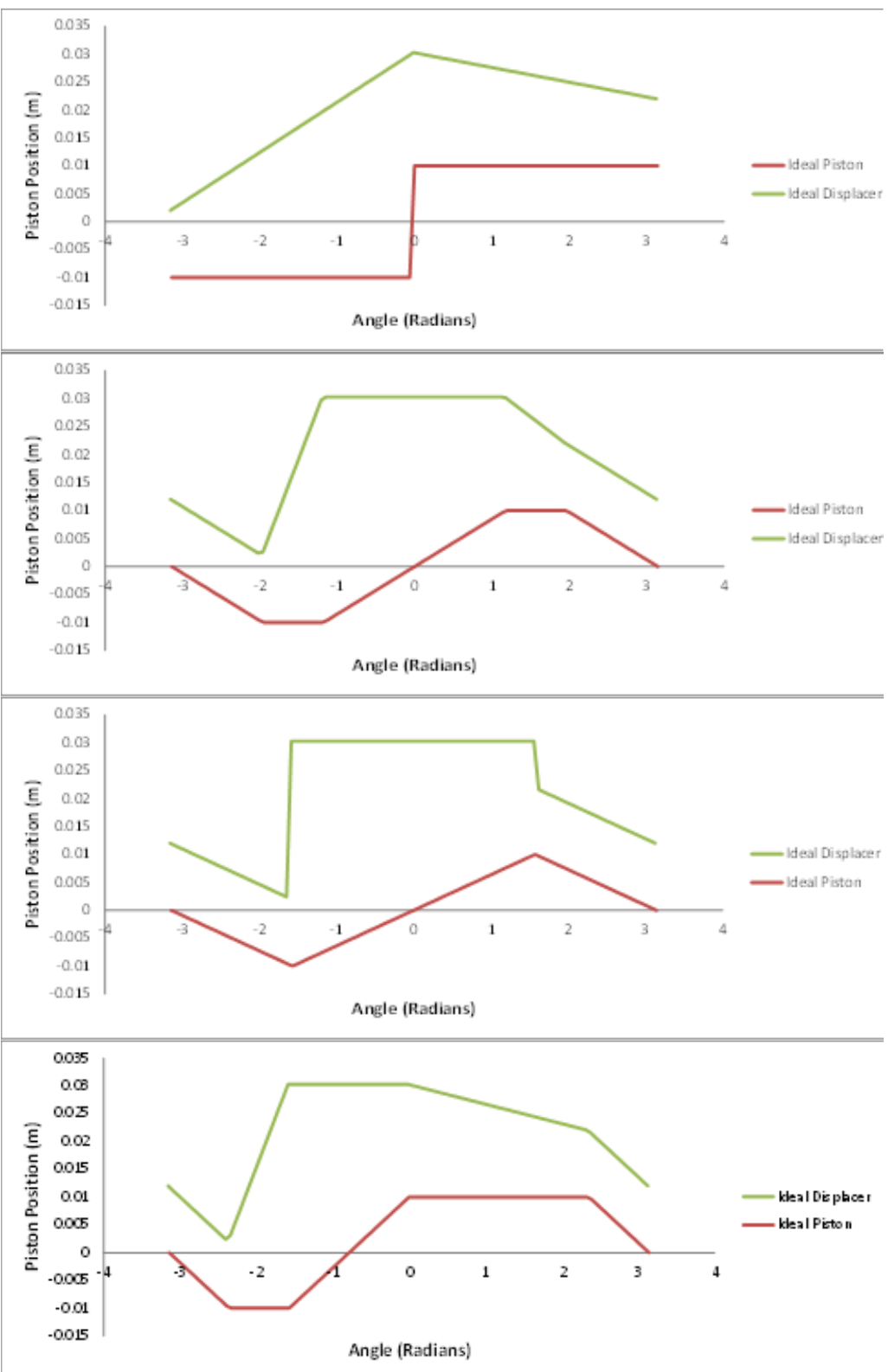

Figure 5. Comparison of four ideal Stirling waveforms

increase pressure drop through the regenerator and heat exchangers, so different versions of the ideal waveform have different power output and efficiency.

In this analysis the piston and displacer are both forced to achieve an ideal waveform. In reality, most freepiston Stirling engines have a free displacer attached to a spring which resonates at the engine operating frequency. These engines have no mechanism to impose a non-sinusoidal waveform on the displacer. However, it is theoretically possible to achieve non-sinusoidal displacer waveforms using displacer alternators and/or non-linear springs. The analysis in this section is intended to show the possible performance benefits of altering piston and displacer motion in engines designed with capability to do so. Engines operating with non-sinusoidal piston and displacer motion are compared to an engine operating with a sinusoidal piston and a free displacer.

\section{A. Ideal Waveforms on Real Engines}

Ideal piston and displacer motion results in maximum specific power and efficiency in ideal Stirling engines. In real engines increased piston and displacer velocities inherent to ideal waveforms can adversely affect engine 
performance by increasing pressure drop through heat exchangers. The following analysis shows that the optimal piston and displacer motions are not necessarily a close approximation of ideal motion, and typically lie somewhere in between the ideal and sinusoidal waveforms.

Analyses of ideal waveforms in this section are done assuming the maximum and minimum working volume, minimum expansion volume, and minimum compression volume to the same values as sinusoidal motion. These constraints limit the inward travel of the piston and displacer leaving a stagnant gas volume in the compression space and limiting the working space stroke. Sage-based nodal analysis of ideal motion under these constraints predicts lower specific power and efficiency than is predicted for sinusoidal motion in many cases.

Since nodal analysis takes into account pressure drop through the heat exchangers and the displacer is not freely moving at the operating frequency the displacer work requirement is non-zero and must be accounted for. Instead of using the working space P-V diagram, which would only accounts for piston work, a combination of instantaneous power and force-displacement (F-D) diagrams for both the piston and displacer are used to show the effect of ideal piston and displacer motion on performance.

Several instances of ideal motion were analyzed using nodal anlaysis including symmetric waveforms of various piston/displacer dwell times and assymettric waveforms focused on minimizing viscous losses. For the sake of brevity, only two cases are presented in this section. Figure 6 shows motion, instantaneous power, and F-D diagrams for both the piston and displacer for a symmetric waveform with a piston dwell time of $\pi / 4$ radians. This motion results in $1680 \mathrm{~W}$ of predicted piston power, compared to $1320 \mathrm{~W}$ predicted for sinusoidal motion. However, short piston dwell times result in high displacer velocities during the cooling phase causing large pressure drops in the heat exchangers and large damping forces on the displacer. This results in $1630 \mathrm{~W}$ of power required to drive the displacer. The increased displacer power not only negates the increase in piston power output, but it nearly negates all of the work done by the piston, so the predicted net power of the engine is reduced to $50 \mathrm{~W}$ compared to $1170 \mathrm{~W}$ predicted for sinusoidal motion. The engine operating under these conditions would produce little more than entropy. 


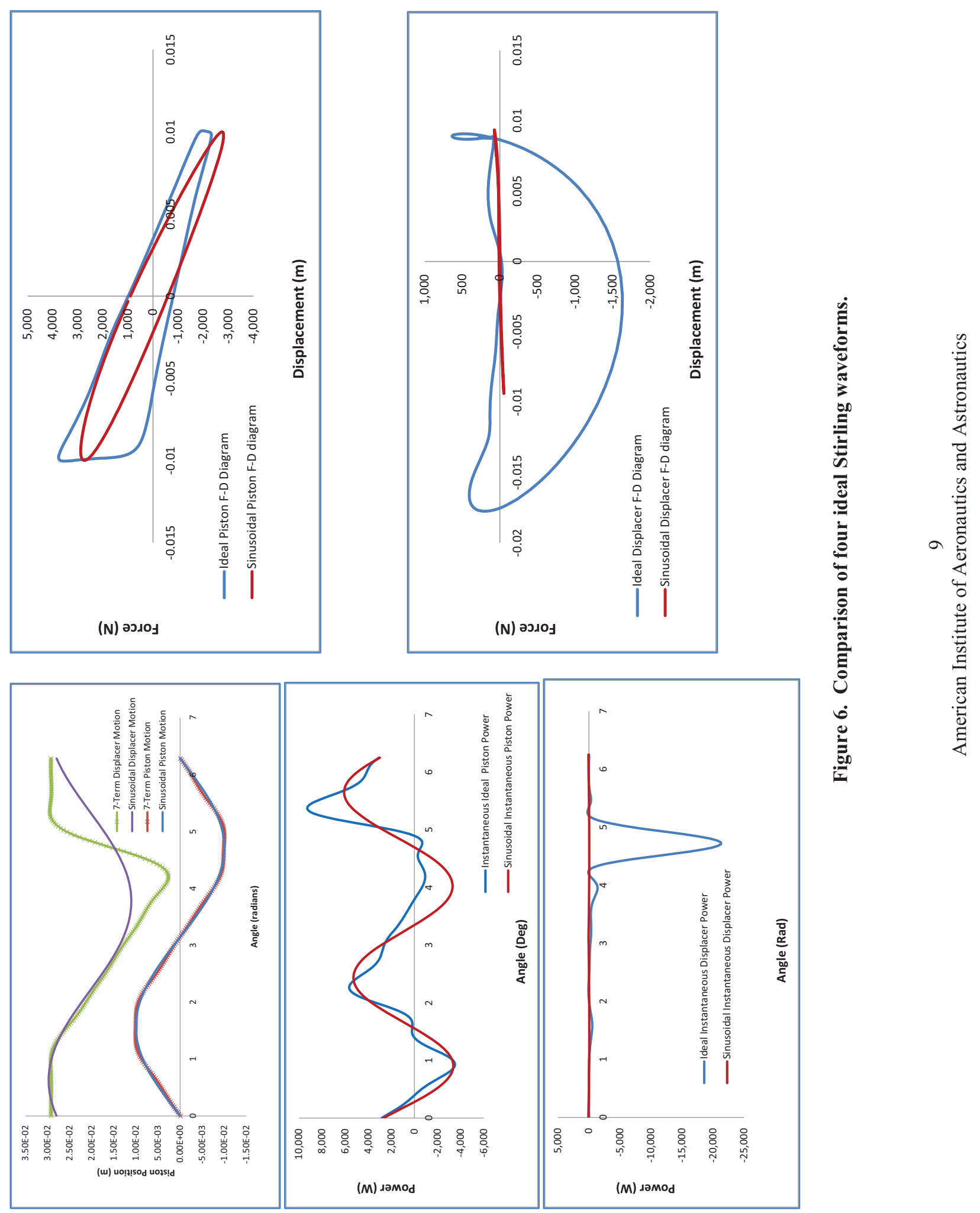


The most successful version of ideal motion run in these analyses was an assymetric waveform chosen to minimize the RMS value of piston/displacer relative velocity, in an effort to minimize pumping losses through the heat exchangers. Figure 7 shows the resulting motion, instantaneous power and F-D diagrams for both the piston and displacer constraining the maximum and minimum working space volume to be the same as an equivalent sinusoidal engine. In this scenario the piston power was $1580 \mathrm{~W}$ with a displacer power requirement of $430 \mathrm{~W}$ resulting in a net power output of $1150 \mathrm{~W}$ at an efficiency of $21.8 \%$. Even in this, the best case scenario for ideal motion, there is no increase in specific power over the sinusoidal case and engine efficiency is reduced.

Nodal analysis shows that ideal waveforms offer no specfic power improvements over sinusoidal waveforms for the geometry and operating conditions analyzed. In both cases piston power increased beyond what was predicted for sinusoidal motion, but parasitic losses, most notably pressure drop through the regenerator, increased as well. The increase in parasitic losses was larger than the increase in piston power, rendering ideal motion useless for this engine.

It should be noted that the results in this section are specific to this engine design and should not be interpreted as broad conclusions. Roughly $90 \%$ of the viscous dissipation in the cases analyzed comes from the regenerator. If other engine designs use less effective regenerators with less pressure drop, or find a method of achieving equal effectiveness with decreased pressure drop, it may be possible for it to benefit from ideal waveforms. 

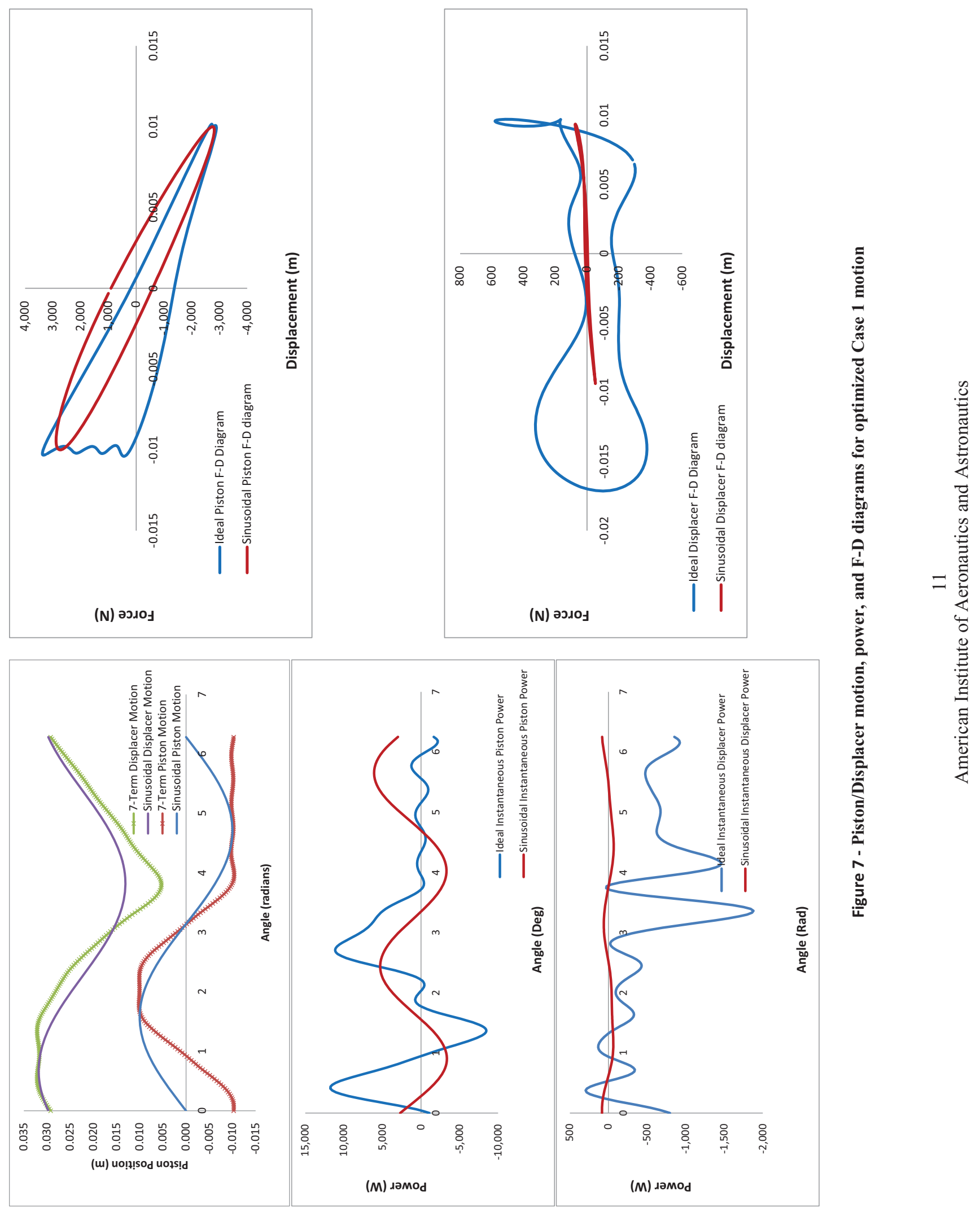


\section{Nodal Analysis of Optimal Waveforms on a Realistic Geometry}

It may seem discouraging that, at least when an ideal engine is constrained to have the same maximum and minimum working space volume as a sinusoidal engine, there is no benefit to achieving ideal motion on the engine chosen for this analysis. However, seeing that ideal motion results in increased piston power in all cases suggests that there may exist some optimal motion that takes advantage of the increased piston power without incurring such a large penalties due to increased viscous losses through the regenerator and heat exchangers. Determining this optimum motion requires the use of non-linear constrained optimization. This was done using the "fmincon" function in Matlab, using net power, as predicted by Sage, as the objective function. In each case the relationship between the optimal waveform and the equivalent sinusoidal waveform are turned into non-linear constraints on the optimization. Independent variables passed to the solver included all seven piston amplitudes, six piston phase angles (the phase angle of the fundamental frequency was pinned to zero), all seven displacer amplitudes and all seven displacer phase angles for a total of 27 independent variables. Using single term sinusoidal motion as the starting point, optimization required hundreds of iterations and thousands of function evaluations. Each function evaluation required a converged Sage solution with enough time nodes to resolve a waveform with 7 harmonics, making the optimization a computationally intensive process.

Figure 8 shows motion, instantaneous power, and F-D diagrams for the piston and displacer for optimized motion with the maximum and minimum working space volumes constrained to be equal to those of the sinusoidal engine. Figure 9 shows the difference between sinusoidal, ideal, and optimal motion under the same constraints. The optimal motion is a compromise between ideal motion and sinusoidal motion, using asymmetric dwell times on the piston to take advantage of increased power and minimizing piston and displacer losses as much as possible by keeping velocities moderate. Optimal motion resulted in $1580 \mathrm{~W}$ of piston power and a displacer power requirement of $150 \mathrm{~W}$, resulting in an increase in net power from $1170 \mathrm{~W}$ to $1430 \mathrm{~W}$ and an efficiency decrease from $30.7 \%$ to $27.9 \%$. Optimal motion under these constraints could potentially offer system level benefits in applications that favor specific power over efficiency. 

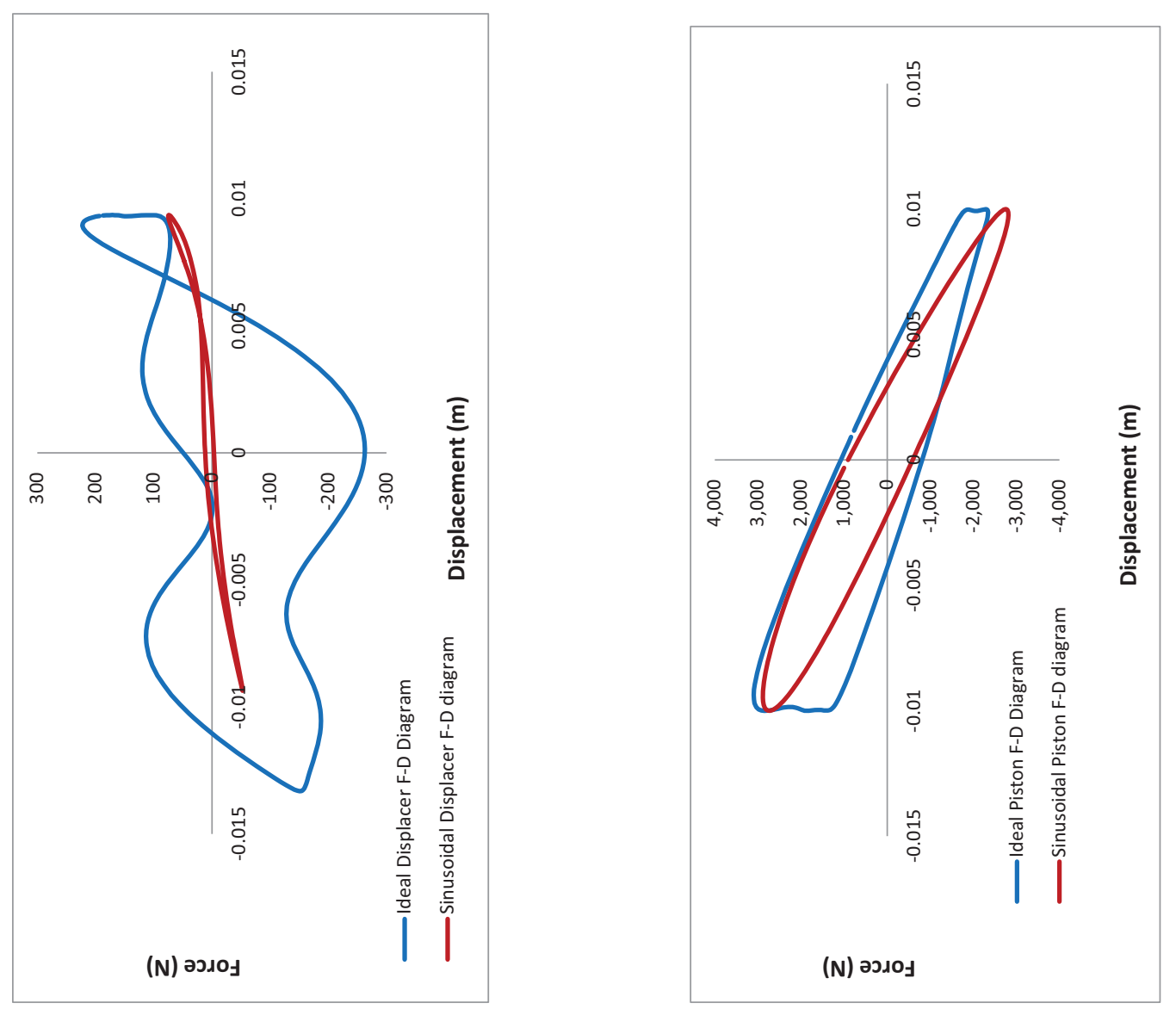

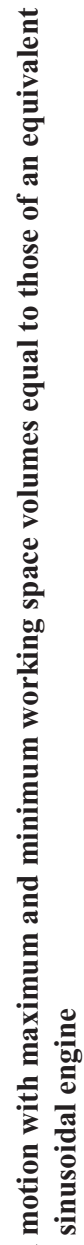
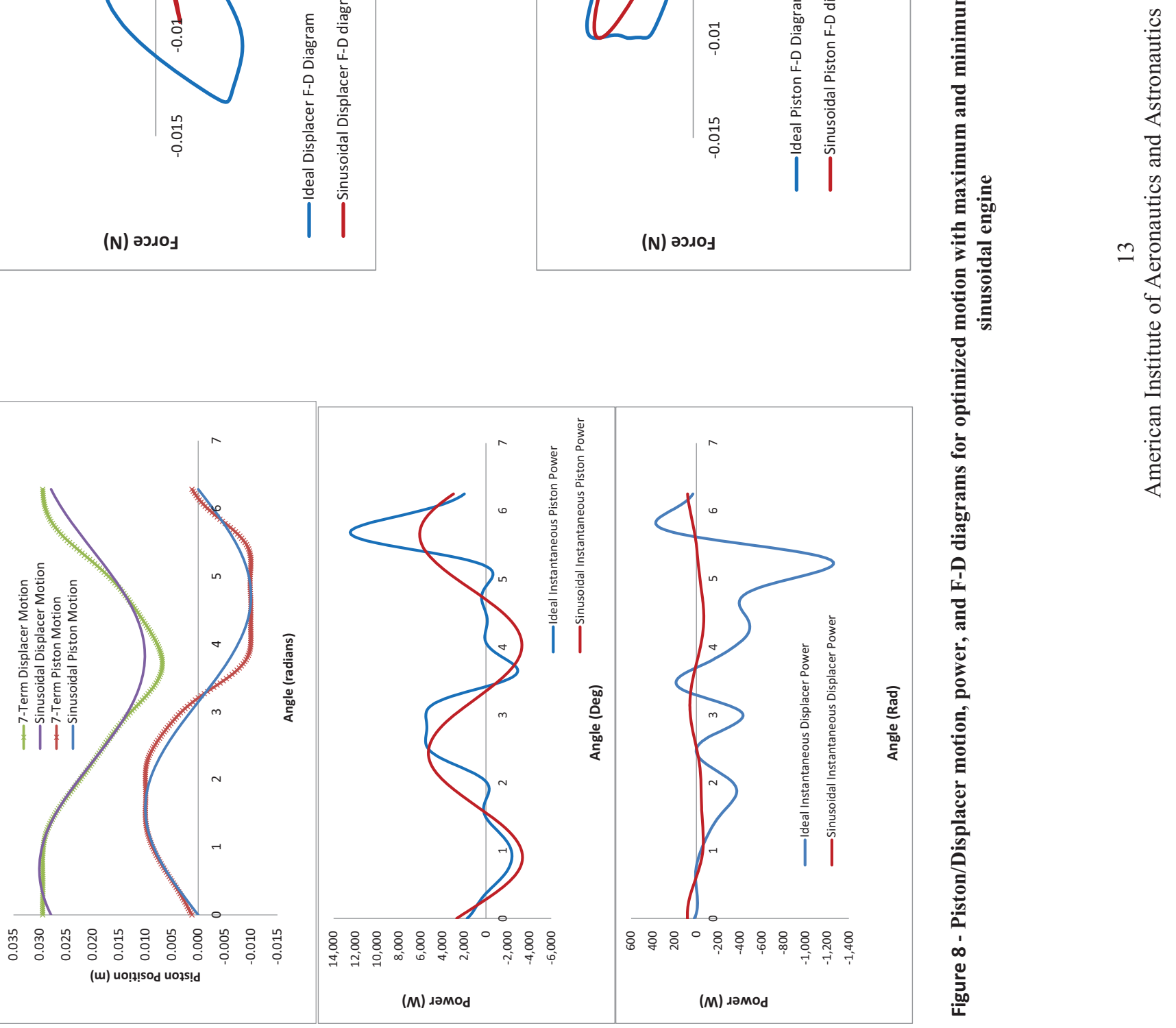


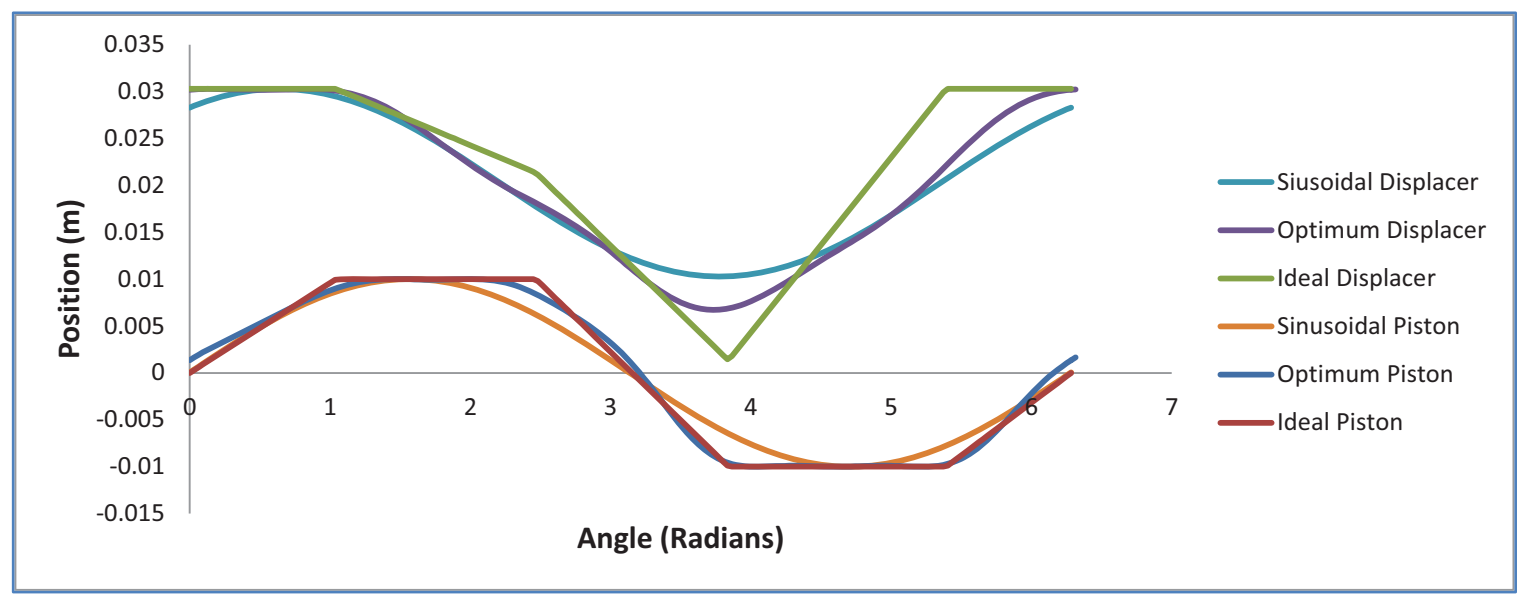

Figure 9. Comparison of sinusoidal, ideal, and optimal piston and displacer waveforms

A less restrictive method of constraining optimized piston displacer motion is to force optimal motion to have the same out-limits of piston and displacer motion and the same pitson-displacer clearance as an equivialent engine using sinusoidal motion. Optimal motion in this case results in a piston power of $1730 \mathrm{~W}$ with $100 \mathrm{~W}$ required to drive the displacer, for a net power of $1630 \mathrm{~W}$ at an efficiency of $26.6 \%$. This is a specific power improvement of $39 \%$ and an efficiency reduction of 13\%. Optimized piston and displacer motion under these constraints could be preferable to sinusoidal motion in applications that give higher weight to specific power.

\section{Concluding Remarks}

This paper consisted of three distinct analyses. First isothermal analysis was used to show that engines using ideal piston waveforms can potentially see large increases in specific power. Next, nodal anlaysis was used to show that these ideal waveforms are not practical in real engines due to increases in parasitic losses, most notably pressure drop through the regenerator. Finally, nodal analysis was combined with non-linear constrained optimization to show that piston/displacer waveforms that are compromises between sinusoidal and ideal waveforms can increase specific power in real engines. The increase in specific power is typically accompanied by a decrease in efficiency, but this could potentially open a trade space for designers in applications that do not rely heavily on efficiency.

The optimum piston and displacer waveforms reported in this paper are specific to the engine chosen for analysis. In general, optimum waveforms are functions of engine geometry, temperature, and pressure gradients within the heat exchangers, and must be determined on a case by case basis. Also, the optimization presented above did not consider efficiency. Designers could perform optimizations with different objective functions, considering both specific power and efficiency, or efficiency alone and arrive at a different solution tailored to their application. There are also several other methods of constraint that could be analyzed, including constraining the out limits of piston and displacer motion to be the same as the sinusoidal case, but removing the constraint on minimum compression space volume or constraining the maximum cycle pressure of the optimal case to equal the maximum cycle pressure of the sinusoidal case.

It should also be noted that this analysis considers only thermodynamic optimization. In order for non-sinusoidal waveforms to provide a real system level benefit the increase in specific power must outweigh losses incurred from whatever drive mechanism is used to achieve those waveforms. A test scheduled to take place at GRC in the Fall of 2014 will attempt to validate the results of the analysis presented herein and quantify drive mechanism losses.

\section{References}

${ }^{1}$ Fission Surface Power Team, "Fission Surface Power Initial Concept Definition," NASA TM-216772, 2010.

${ }^{2}$ Walker, G., Stirling Engines, Clarendon Press, Oxford, 1980, Chaps. 1-4. 